\title{
From the Editors' Desk: The Power of First Impressions
}

\author{
Richard L. Kravitz, MD, MSPH \\ Division of General Medicine, University of California at Davis, Sacramento, CA, USA.
}

$I^{n}$ n Blink: The Power of Thinking without Thinking (Little, Brown \& Co. 2005), the journalist Malcolm Gladwell writes about the way first impressions-judgments reached within two minutes of encountering a person, thing, or situation-can yield remarkably accurate results. At the same time, he warns about how such "thin-slicing" can readily be hijacked by prejudice. So, for example, watching just a few seconds of interaction between a married couple (with attention to tone of voice, facial expressions, and gestures) predicts the future health of their marriage, while misreading the intentions of a criminal suspect like Amadou Diallo can lead to tragic results.

In this issue of JGIM, two articles illustrate the power of thinslicing in medicine. In the first, Hardy et al. show how a simple measure of self-reported functional performance (ability to walk a quarter-mile) predicts future functional status, hospitalization rates, and health care costs. The findings are reminiscent of a meta-analysis published in these pages (DeSalvo et al., JGIM, March 2005) which showed a strong relationship between single-item health status and mortality. In a sense, such work is part of a recurring narrative in health measurement, in which clinical impressions are replaced by complex scales with good psychometric properties, which in turn evolve into shorter and shorter measures with greater clinical and practical utility. Thus the Medical Outcomes Study self-reported health scales begat the SF-36, which begat the $\mathrm{SF}-12$, which begat the SF-1. Shorter measures trade off psychometric rigor for greater ease of implementation; ironically, in their brevity, the ultra-short measures start to resemble the traditional clinical interview questions their psychometric progenitors displaced.

The second article in the spirit of Blink comes to us from Hwang et al. Their paper describes the results of a study in which 58 physicians rated 126 patient photographs as looking older or younger than their "stated age." Being rated as 5 or more years older than stated (actual) age was associated with poor patient self-reported health, defined as $>=2$ standard deviations below the mean on SF-12 physical or mental health composites. The sensitivity of this appearance-based clinical impression was poor (29\%), but the specificity was moderate (82\%). In short, first impressions convey useful information. However, they cannot be fully relied upon and should be confirmed via careful clinical investigation.

Also in this issue of JGIM are several articles related to screening for cancer and mental health conditions, including pieces concerning what physicians know about colorectal cancer screening (Yabroff et al.: not that much), how breast cancer screening and follow-up can be improved (Donelan et al. and Philips et al.: through patient navigators; Atlas et al.: through intelligent use of information systems), and whether alcohol screening in the inpatient setting is worthwhile (Bradley et al.: it depends).

The line separating thin-slice impressionism from bias and prejudice is itself pretty thin. The key to appropriate application of first impressions is to subject them to care scientific evaluation. Do the articles in this issue succeed in doing this? We encourage readers to share their reactions.

Richard L. Kravitz, MD, MSPH, UC Davis Division of General Medicine, 4150 V. Street, Suite 2400 PSSB, Sacramento, CA 95817 , USA; (e-mail: rlkravitz@ucdavis.edu).

J Gen Intern Med 26(2): 103

DOI: $10.1007 / \mathrm{s} 11606-010-1595-3$

(C) The Author(s) 2010. This article is published with open access at Springerlink.com

Open Access: This article is distributed under the terms of the Creative Commons Attribution Noncommercial License which permits any noncommercial use, distribution, and reproduction in any medium, provided the original author(s) and source are credited.

Corresponding Author: Richard L. Kravitz, MD, MSPH, UC Davis Division of General Medicine, 4150 V. Street, Suite 2400 PSSB, Sacramento, CA 95817, USA (e-mail: rlkravitz@ucdavis.edu). 